\title{
Introductory notes for the Acta IMEKO Special Section on the Asia-Pacific Symposium on Measurement of Mass, Force and Torque (APMF 2017)
}

\author{
Rugkanawan Wongpithayadisai ${ }^{1}$, Jeerasak Pitakarnnop ${ }^{1,2}$ \\ ${ }^{1}$ National Institute of Metrology, Pathumthani, Thailand \\ ${ }^{2}$ Aerospace Engineering, Chulalongkorn University, Bangkok, Thailand
}

\begin{abstract}
Section: Editorial
Citation: Rugkanawan Wongpithayadisai, Jeerasak Pitakarnnop, Introductory notes for the Acta IMEKO Special Section on the Asia-Pacific Symposium on Measurement of Mass, Force and Torque (APMF 2017), Acta IMEKO, vol. 8, no. 3, article 1, September 2019, identifier: IMEKO-ACTA-08 (2019)-03-01
\end{abstract}

Editor: Dušan Agrež, University of Ljubljana, Slovenia

Received: June 11, 2019; In final form September 30, 2019; Published September 2019

Copyright: This is an open-access article distributed under the terms of the Creative Commons Attribution 3.0 License, which permits unrestricted use, distribution, and reproduction in any medium, provided the original author and source are credited

Corresponding author: Rugkanawan Wongpithayadisai, e-mail: rugkanawan@nimt.or.th

\section{Distinguished Readers,}

This special issue of Acta IMEKO is the second issue, which presents the research highlights of the biannual Asia-Pacific Symposium on Measurement of Mass, Force and Torque (APMF). Over the past two decades since its first organizing in 1992, the APMF symposium cosponsored by IMEKO TC3 has developed into one of the most active symposia in the AsiaPacific region. The recent APMF was organized by National Institute of Metrology (Thailand) in November 2017 in Krabi, Thailand, aka the Land of Smiles, around the theme "Metrology Moving Towards Foundation". The fields of the discussions and presentations were extended to cover the area of mass, force, torque, density, hardness, pressure, vacuum and gravimetry, and consequently the future formal name "Asia-Pacific Measurement Forum on Mechanical Quantities (APMF)". With the contributions from 62 participants from 11 countries, 47 scientific papers were presented with a fruitful outcome. The International Program Committee of APMF 2017 considered and selected 10 potential papers to be published as the enhanced versions in Acta IMEKO journal. With the journal's standards of the review process by the expert referees, 6 articles were accepted for this special issue.

In the field of mass metrology, the state-of-the-art strategy for calibrating non-automatic weighing instruments based on EURAMET cg-18 was precisely and clearly summarized with the explanation of its scientific principle by Klaus Fritsch. Differing from the existing national guidelines, EURAMET cg-18 suggested the uncertainty of a weighing result that described the device performance during day-to-day usage not only at the time of calibration. To achieve the required accuracy complying with the quality of weighing process, the prerequisite concept of minimum weight under consideration of safety factor was introduced and described.

There was the continuous development of small force metrology by NIM, China. A $10 \mathrm{~N}$ small force standard machine with the air bearing support was proposed and investigated its performance by Gang $\mathrm{Hu}$. The machine's minimum force was extended to $1 \mathrm{mN}$ to serve the demands of force measurements in the range of millinewton to newton, e.g., biology applications, pharmaceutical industries, nano-hardness metrology and precision manufacturing. The mechanical system's structure and main components of the machine were newly designed to reduce the standard uncertainties, and consequently achieved the higher accuracy of force measurement in this small range.

Two interesting papers from NMIJ/AIST were presented in the field of pressure metrology. Hiroaki Kajikawa studied the long-term drift characteristics of three kinds of hydraulic pressure gauges (different sensing structures). The results from the experimental investigation led to the practical way to reduce and compensate the long-term drift, i.e., the application of preliminary pressure that was very close to the measurement pressure. Furthermore, the drift could also be corrected by tracing a zero reading during the pressure application. Hideaki Iizumi proposed the solution to achieve the calibration values of high pressure unaffected by the kind of gas medium and the setting posture of quartz Bourdon-type pressure transducers. Extending from his recent study in which the analysis was carried 
out with the horizontally mounted transducers, the vertically mouthed transducers were investigated. This concept is very useful for the transfer standards used for the international comparisons.

NIMT's big project of torque realization up to $5 \mathrm{kN} \cdot \mathrm{m}$ of torque standard machine was conducted. Nittaya Arksonnarong presented the principle of the designs and the performance of the developed machine. The flexure bearing was selected as the fulcrum to improve the machine's strength and to shorten the stabilizing time of the lever arm's swing. Less maintenance and lower construction costs than the machine using air bearing are the dominant advantages of this design. The machine's capability and its claimed CMCs at $0.01 \%(k=2)$ had been confirmed by the satisfactory results from the informal comparison with the recognized NMI, PTB.

Last but not least, another project in the torque metrology was proposed by Nattapon Saenkum. NIMT continuously attempted to improve the performance of its elastic hinge type torque standard machine. The concept of the separate calibrations of the signals observed at the left hinge, the right hinge and the fulcrum, was introduced to deal with the undesirable influences from the bending moment by obtaining the residual torque. With the corrections of the residual torque value and the difference arm length, the torque characteristics, i.e., the repeatability, the reproducibility and the linearity were significantly improved, and consequently the better CMC at $0.020 \%(k=2)$ at the measurement range $2 \mathrm{Nm}$ to $10 \mathrm{Nm}$.

It is our extreme honour to serve as the Section Editors for this knowledgeable issue of this open-access journal. We extend heartfelt thanks to the authors, reviewers, associated editor, copy editor, layout editor and article editor for their supports and dedications to this issue. The publication of this special issue would not be possible without the great supports from Dr. Rolf Kumme, the IMEKO TC3 Chairperson and Dr. Yon Kyu Park, the IMEKO TC3 Vice Chairperson. Our huge gratitude goes to Dr. Dirk Röske, who always provided his prompt assistances and advices on the journal's online system. We would like to express our appreciations to Dr. Min-Seok Kim, the former section editor of the special issue of the APMF 2015 for sharing his experience of being journal's section editor. We fully appreciate Prof. Dr. Dušan Agrež, the Editor in Chief, for his kind contribution to the issue. Lastly, we hope you enjoy reading our work.

\author{
Rugkanawan Wongpithayadisai \\ Jeerasak Pitakarnnop \\ Section Editors
}

\section{Editorial to additional papers}

\section{Dear Reader,}

Five additional papers are closing the third issue of Acta IMEKO in 2019.

In the paper submitted by Saher R. Hassan et al. is presented investigation of the influence of the shaker armature's mechanical structure on periodic force measurement, with emphasis on measurement uncertainty. In order to perform modal and harmonic analyses, a finite element model was iteratively developed. The results of validation show that the dynamic behaviour of the shaker armature should be taken into consideration before carrying out a periodic force measurement.

Andreas Brüge et al. present the realisation and dissemination of the unit of rotatory power at the Physikalisch-Technische Bundesanstalt (PTB) aimed at ensuring the safe operation of ergometers for patients. This task is carried out by means of a standard that can be operated both as a regulated brake and as a regulated drive, so that the effective torque and the revolution speed can be measured traceably. At the end, technical details and uncertainty calculations about this standard are given.

Gisa Foyer et al. analyse the torque measurement in the $\mathrm{MN} \cdot \mathrm{m}$ range. It is important in nacelle test benches, as it constitutes the primary value for the efficiency determination of wind turbine drive trains. However, no traceable measurement is possible above $1.1 \mathrm{MN} \cdot \mathrm{m}$. Several torque measurement methods are presented and discussed with respect to traceability and measurement uncertainty. It was found that these methods cannot fully substitute direct measurements. Therefore, detailed recommendations for a torque calibration procedure based on the specific conditions in nacelle test benches are given so as to enable the traceability of torque measurement using a transfer standard.

Alessandro Gallo et al. are focused on models of submerged structures and underwater archaeological finds, which environments are characterised by poor visibility conditions. Performance analysis has been conducted on a multi-view technique in comparison with different image enhancement algorithms that are commonly used in air in order to highlight their limits in the underwater environment. The results have been adopted to reconstruct an area of $40 \mathrm{~m}^{2}$ at a depth of about $5 \mathrm{~m}$ at the underwater archaeological site of Baiae (Italy).

The aim of the paper submitted by Sebastian Baumgarten et al. is to deal with torque measurements. In torque standard machines, different kinds of bearings are used to reduce the influence of parasitic loads under the assumption that such loads are low. In this article, the parasitic loads of the torque standard machines at the PTB, Teknologian tutkimuskeskus VTT Oy (VTT), and Cesky Metrologicky Institut (CMI) were characterised and compared. The results are presented together with a proposal for an evaluation.

Have a fruitful reading of this interesting issue of Acta IMEKO.

Dušan Agrež, Editor-in-Chief of Acta IMEKO 